\title{
Successful retrieval of a dislodged Zotarolimus-eluting coronary stent in the ascending aorta
}

\begin{abstract}
Coronary stent dislodgment or embolization is rare in the era of 3rd generation Drug Eluting Stents but is a challenging complication in interventional cardiology. Intracoronary embolization of the dislodged stent is associated with a high risk of coronary occlusion, due to thrombus formation and subsequent myocardial infarction. Furthermore, systemic embolization may cause severe cerebrovascular events. Nonsurgical retrieval options for this complication are being used with good results in most cases, but bailout cardiac surgery may be indicated if percutaneous retrieval attempts fail. We present here a case of dislodgement of a zotarolimus-eluting coronary stent during percutaneous coronary intervention and successful retrieval from the ascending aorta using a Amplatz goose neck microsnare.
\end{abstract}

Keywords: Coronary stent dislodgment - Zotarolimus-eluting coronary stent - Amplatz goose neck microsnare $\boldsymbol{*}$ Percutaneous coronary intervention

\section{Introduction}

Coronary stent dislodgement is a rare but known complication of percutaneous coronary intervention [1]. Stent loss has been associated with severe complications, including embolization to the coronary circulation, embolic cerebrovascular events and peripheral embolization. Retrieval of a dislodged stent can be done either percutaneously or surgically [2]. We report a rare case of stent dislodgement in the ascending thoracic aorta during percutaneous coronary intervention to a calcified proximal left circumflex artery (LCX) lesion.

\section{Case Report}

A 78-year-old hypertensive and diabetic gentleman was admitted to our hospital with a history of exertional angina since 3 months and past history of percutaneous coronary intervention (PCI) to LAD 4 years back. Coronary angiography showed calcified major coronaries, patent LAD stent with $80 \%$ diffuse lesion of proximal left circumflex artery extending into Obtuse marginal (Figure 1a) and normal RCA. Patient was taken up for PCI to LCX. 6Fr XB (Xtra backup/3.5, Cordis Corporation) guiding catheter was used to engage the left main via right femoral approach. The lesion was crossed with a 0.014 " Fielder FC (ASAHI) guide wire. Lesion was serially dilated with a $1.25 \times 10,1.5 \times 10,2.0 \times 10$ balloons (Figure 1b). After what was thought to be an adequate dilatation, a $2.5 \times 30 \mathrm{~mm}$ zotarolimus-eluting coronary stent (Resolute integrity, Medtronic, Inc) was tried to pass across the lesion. There was considerable resistance for the stent delivery. The lesions were again dilated with a $2.5 \times 10 \mathrm{~mm}$ balloon and another 0.014 " guiding wire was used as support. However, again stent had significant resistance to cross through the lesion (Figure 2a). The stent was again retracted and rotablation was planned to have adequate bed preparation. However on retracting the stent catheter, we were surprised to see only the balloon without stent. A search for the dislodged stent was made under fluoro guidance. As the dislodged stent could not be found in the coronaries or anywhere on the guidewire it was thought to be somewhere inside the guiding catheter. Hence the whole system was removed insitu and a search was made inside the guiding catheter. But however
Adithya Udupa* Apollo Hospital, Mysore, Karnataka, India

*Author for correspondence:

Tel.: +7506242952

udupaputti@gmail.com

Submitted: 10 June 2017

Accepted: 29 January 2018

Published online: 05 February 2018 


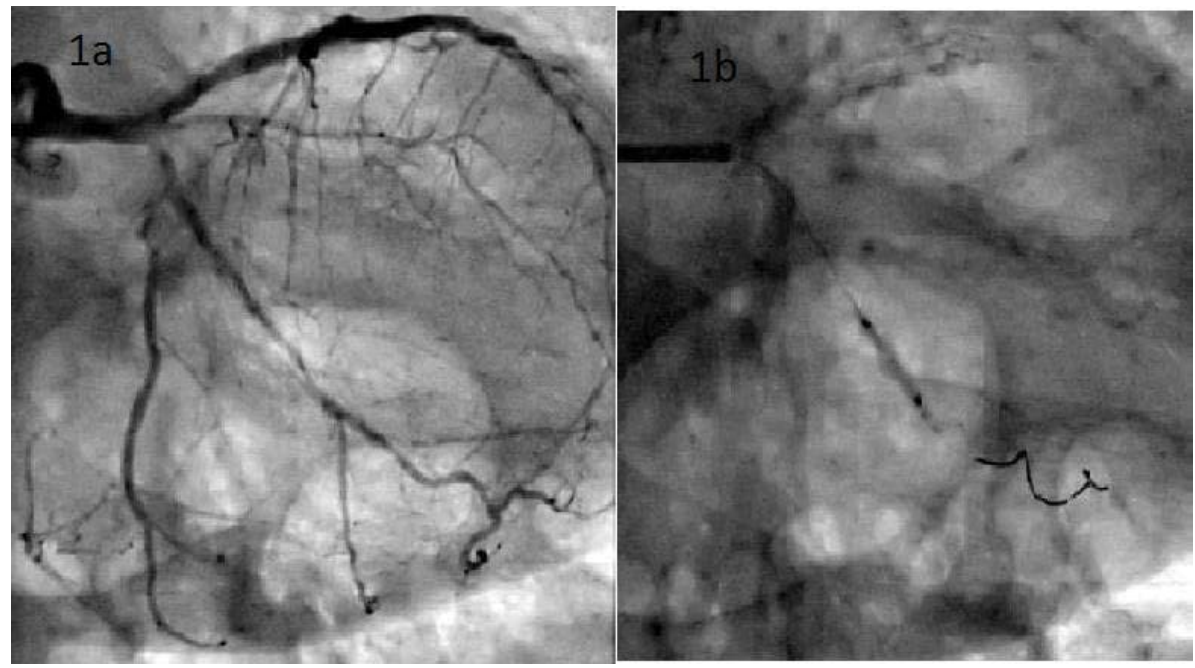

Figure 1: a) RAO caudal view showing diffuse calcified lesion of LCX and OM. b) showing predilation of the lesion in same view.

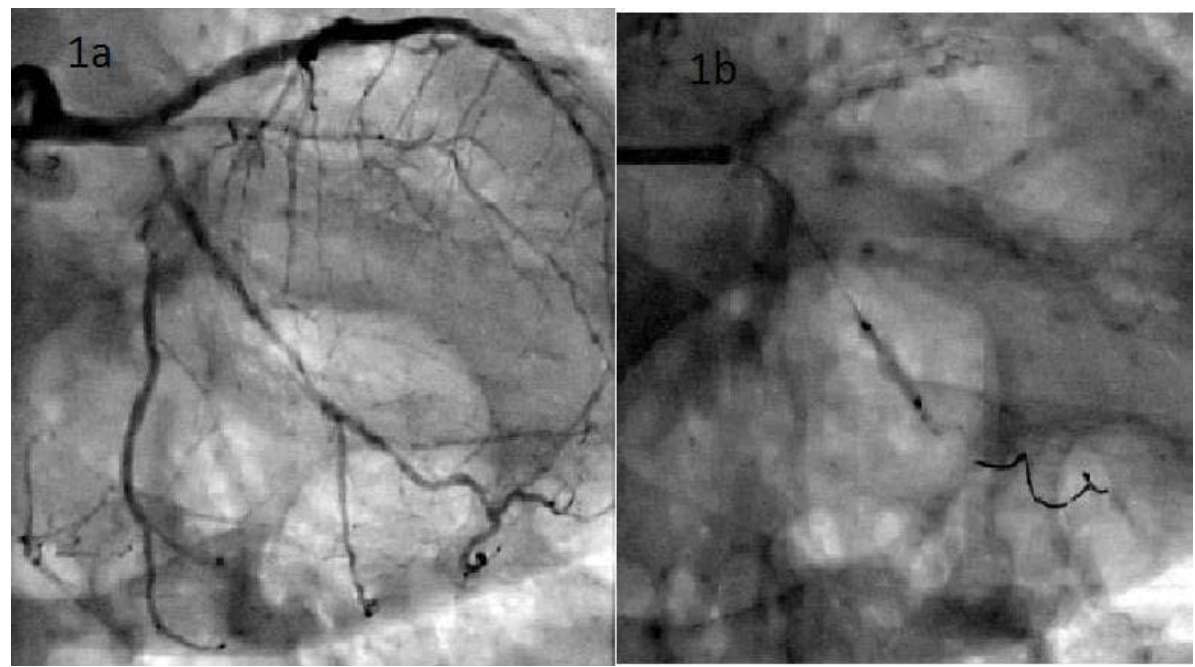

Figure 2: a) RAO caudal view showing guide catheter buckling up on attempt to stent deployment. b) Showing dislodged stent freely floating in ascending aorta in AP view, vertical arrow head showing fixation of proximal end to LCX ostia and horizontal arrow head pointing the distal floating edge.

there were no signs of the dislodged stent inside the guiding catheter or guiding wire.

Again a renewed effort was made to search for the dislodged stent under double magnification in various angles. Now we could see the stent hanging precariously, unwound extending from the proximal LCX and into the ascending aorta (Figure 2b). An Amplatz Goose neck Microsnare was pased through the XB guiding catheter and an attempt to grasp the stent was made, unsuccessfully. Another attempt with same snare was tried using a Judkins right guiding catheter. Now the snare was able to grasp the floating stent (Figure 3a) and with a mild tug the stent was freed from the calcified LCX and was able to be retrieved (Figure 3b). Patient remained hemodynamically stable throughout the procedure.

\section{Discussion}

Stent dislodgement is a rare complication that has been reported in $0.32-8.4 \%$ of PCI procedures [1]. The incidence of stent loss has been decreasing in recent times primarily due to technological advances in PCI and stent design. Brilakis et al., [3] reported that the annual incidence of stent loss during PCI in a single center, increased from 1994 to 1997, but after that subsequently declined. However, stent dislodgement is still a potential cause of serious complications such as death, stroke, embolization, and need for an emergency operation [3].

Dislodgement of a stent can be secondary to extreme coronary angulation, highly calcified coronary arteries, inadequate bed preparation, and direct stenting [4]. Thus, adequate bed preparation with predilation or 

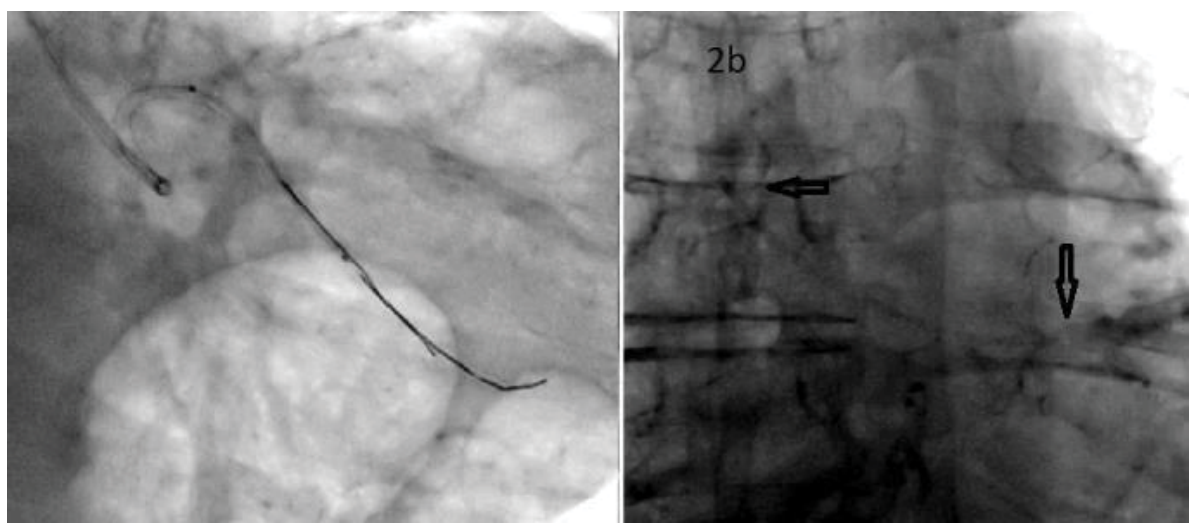

Figure 3: a) showing floating edge being captured by the snare and retrieving into JR guiding (arrow head), b) the photograph of retrieved stent along with the snare.

rotablation may help prevent stent loss. In our case, the stent dislodgement was most probably secondary to the highly calcified LCX, angulation and possible inadequate coronary artery predilation. A lower threshold for rotablation could have possibly prevented this complication.

Numbers of techniques have been described for retrieval of dislodged coronary stent like, a small balloon technique, a double wire technique, or a loop snare. The choice of retrieval device and technique usually depends on the location of the stent, the type of stent, its deployment status and operator experience. A snare loop is relatively safe and easy to use. It has a low rate of complications and appears to be effective [5].A snare loop appeared to be the best method of retrieval in our case, as free edge of lost stent was hanging in the ascending thoracic aorta.

Nowadays, stent loss and dislodgement occur rarely. However, every catheterization laboratory should be equipped with instruments for intravascular foreign body retrieval and interventional cardiologists should be familiar with these retrieval methods and techniques.

\section{Executive summary}

Coronary stent dislodgment or embolization is rare in the era of 3rd generation Drug Eluting Stents but is a challenging complication in interventional cardiology.

Intracoronary embolization of the dislodged stent is associated with a high risk of coronary occlusion, due to thrombus formation and subsequent myocardial infarction. Furthermore, systemic embolization may cause severe cerebrovascular events. Nonsurgical retrieval options for this complication are being used with good results in most cases, but bailout cardiac surgery may be indicated if percutaneous retrieval attempts fail.

We present here a case of dislodgement of a zotarolimus-eluting coronary stent during percutaneous coronary intervention and successful retrieval from the ascending aorta using a Amplatz goose neck microsnare.

\section{References}

1. Eggebrecht $\mathrm{H}$, Haude M, von Birgelen C, et al. Nonsurgical retrieval of embolized coronary stents. Catheter. Cardiovasc. Interv. 51: 432-440 (2000).

2. Yang SC, Chong E, Sangiorgi GM. A challenging case of dislodged stent retrieval with the use of Goose neck snare kit. Catheter. Cardiovasc. Interv. 75: 630-633 (2010).

3. Brilakis ES, Best PJ, Elesber AA, et al. Incidence, retrieval methods, and outcomes of stent loss during percutaneous coronary intervention: a large single-center experience. Catheter. Cardiovasc. Interv. 66: 333-340 (2005).

4. Nikolsky E, Gruberg L, Pechersky S, et al. Stent deployment failure: reasons, implications, and short-and long-term outcomes. Catheter. Cardiovasc. Interv. 59: 324-328 (2003).

5. Koseoglu K, Parildar M, Oran I, Memis A. Retrieval of intravascular foreign bodies with goose neck snare. Eur. J. Radiol. 49: 281-285 (2004). 